\title{
A INFLUÊNCIA DA MARCAÇÃO DO PRIMEIRO GOL NO RESULTADO FINAL DE PARTIDAS DO CAMPEONATO BRASILEIRO SÉRIES A E B ${ }^{1}$
}

DOI: $105902 / 0102830819261$

Data de submissão: 27-08-2015

Data de Aceite: 21-10-2016

Victor Alberice de Oliveira Rodrigues

Universidade Federal de Minas Gerais valberice@gmail.com

Frederico Gonçalves Constantino

Universidade Federal de Minas Gerais

constantino.frederico@gmail.com

Gibson Moreira Praça

Universidade Federal dos Vales do Jequitinhonha e Mucuri gibson_moreira@yahoo.com.br

Cristino Julio Alves da Silva Matias Universidade Federal de Minas Gerais crismatias@gmail.com

Pablo Juan Greco

Universidade Federal de Minas Gerais grecopj@ufmg.br

Resumo: Estudo objetivou analisar a influência de se marcar o primeiro gol da partida no resultado final do Campeonato Brasileiro séries A e B. Analisou-se o resultado final e a marcação do primeiro gol de jogos 2011 a 2014 nas séries A e B. Nos resultados observouse associação significativa entre marcar o primeiro gol e vencer as partidas na série $A$. Resultados similares foram observados na série $B$, revelando a importância da marcação do primeiro gol para a obtenção de vitórias em partidas de futebol. Conclui-se haver uma associação significativa entre marcar o primeiro gol e vencer no campeonato brasileiro.

Palavras-chave: Futebol; Variáveis Situacionais; Campeonato Brasileiro

'Subsídio financeiro da Fundação de Amparo à Pesquisa do Estado de Minas Gerais (FAPEMIG) e Coordenação de Aperfeiçoamento de Pessoal de Nivel Superior (CAPES). 


\section{Introdução}

O jogo de Futebol é aleatório, imprevisível, dinâmico (GARGANTA, 1997), e demanda dos jogadores uma adequada interação dos componentes do rendimento esportivo relacionados às capacidades físicas, técnicas, táticas e psicológicas (AGUIAR et al., 2012). A análise individual e da equipe é essencial para melhorar o desempenho no futebol (CARLING; WILLIAMS; REILLY, 2002), assim é comum no futebol a utilização de indicadores de desempenho, que são definidos como a seleção e a combinação de variáveis, o que ajudam a fornecer feedback e a alcançar o sucesso (CARLING et al., 2005; HUGHNES; BARTLETT, 2002).

O sucesso de uma equipe no jogo de futebol é determinado pelo número de gols marcados e sofridos (GOMES; SOUZA, 2008) deste modo as equipes devem desenvolver meios eficazes para ganhar a bola, criar ataques bem-sucedidos, oportunizar chances eficazes para fazer gols e completá-las marcando gols com uma alta eficiência (LUHTANEN et al., 2001). Assim dentre os muitos comportamentos técnicos e táticos, aqueles que conduzem aos gols são os mais estudados (LEITE, 2014).

O resultado de um jogo de futebol é o final de uma sucessão de ações ou comportamentos compostos por decisões e execuções, individuais e interações coletivas, suportadas ou não pelo de modelo de jogo da equipe (GARGANTA, 1997; SILVA, 2008). Deste modo, o resultado de um jogo suporta-se numa lógica complexa de acontecimentos de certa forma previsíveis e imprevisíveis (ARAÚJO et al., 2006). Assim, um acontecimento marcante, como um gol, representa a assunção de novas relações entre os componentes do sistema "jogo", que se organizam em função de conseguir um empate/manter a vitória.

Apesar do fato de que há uma ampla quantidade de estudos que analisaram as características dos gols que têm sido marcados em vários torneios (GARGANTA, 1997; MICHAILIDIS et al., 2004; YIANNAKOS;ARMATAS, 2006) a necessidade de constante registro e avaliação das características de futebol se torna importante, pois este apresenta uma contínua evolução na solicitação de rendimento ou evolução técnicatática (YIANNAKOS;ARMATAS, 2006). Entender esse processo é essencial para nortear a planificação e preparo das equipes. A influência do primeiro gol na partida tem sido estudada 
tanto no futsal, versão indoor do futebol (LEITE, 2014), quanto no futebol. Michailidis et al. (2013) apontaram que na Copa dos Campeões Europeus de 2012 as equipes que marcaram o primeiro gol da partida venceram o jogo 70.97\% das vezes. Monlinuevo e Bermejo (2012) analisaram a influência da marcação do primeiro gol na liga espanhola de futebol e na liga de futsal. Em ambas ligas se observou uma vantagem na obtenção da vitória para as equipes que marcaram o primeiro gol da partida. Esses resultados apresentaram uma alta associação entre a marcação do primeiro gol e a vitória. Contudo, a influência da marcação do primeiro gol em partidas do campeonato brasileiro ainda é desconhecida, lacuna que se torna ainda mais evidente quando buscam-se estudos que tenham, concomitantemente, analisado dados - não somente em relação à marcação do primeiro gol -relativos às diferentes divisões de campeonatos nacionais (CASTELLANO; CASAMICHANA, 2015). Assim este estudo objetivou investigar a associação entre a marcação do primeiro gol e o resultado final de jogos do Campeonato Brasileiro séries A e B.

\section{Métodos}

Este estudo possui caráter descritivo (THOMAS et al., 2012), cujo objetivo é apresentar aportes iniciais acerca de um fenômeno.

Para análise da influência do primeiro gol, coletaram-se dados de jogos dos campeonatos brasileiros dos anos de 2011, 2012, 2013 e 2014 a partir do site www.ogol.com.br. Os dados foram organizados e tabulados com recurso ao software Excel for Windows 2013. Para a análise inferencial, recorreu-se ao teste de qui-quadrado de associação, mantendo-se um nível de significância de 5\%. O software SPSS 20.0 foi adotado para as análises.

\section{Resultados}

As tabelas 1 e 2 apresentam a associação entre a marcação do primeiro gol e o resultado final das partidas (respectivamente para a série A e série B). Dados são expressos em termos da incidência absoluta de resultados em cada uma das combinações possíveis entre o resultado do jogo (vencer ou não vencer) e marcação do primeiro gol (sim ou não). 
Tabela 1: Serie A

\begin{tabular}{lllllcl}
\hline & $\mathrm{P} / \mathrm{P}$ & $\mathrm{P} / \mathrm{nV}$ & $\mathrm{nP} / \mathrm{n}$ & $\mathrm{nP} / \mathrm{nV}$ & Qui-quadrado & Valor de $\mathrm{p}$ \\
\hline $\mathbf{2 0 1 1}$ & 223 & 123 & 45 & 303 & 194,276 & $0,001^{*}$ \\
$\mathbf{2 0 1 2}$ & 172 & 120 & 55 & 343 & 123,675 & $0,001^{*}$ \\
$\mathbf{2 0 1 3}$ & 231 & 106 & 34 & 303 & 238,419 & $0,001^{*}$ \\
$\mathbf{2 0 1 4}$ & 210 & 139 & 139 & 207 & 28,803 & $0,001^{*}$ \\
\hline
\end{tabular}

Legenda: $\mathrm{P}=$ marcou primeiro gol; $\mathrm{nP}=$ Não marcou primeiro gol; $\mathrm{V}=$ Venceu; $\mathrm{nV}=$ Não Venceu.

$\mathrm{Na}$ Tabela 1 apresentam-se os resultados dos jogos dos Campeonatos Brasileiros da Serie A nos anos de 2011 a 2014. Observou-se a partir do teste Qui-quadrado, uma associação significativa entre marcar o primeiro gol e sair vencedor na partida nesta serie em todos os anos analisados ( $p=0,001$ todos os anos). Ainda na Tabela 1 nota-se que nos anos de 2011 a 2013 o parâmetro nP/nV obteve a maior incidência, onde no ano de 2014 foi superada pelo parâmetro P/V. De forma inversa observa-se que em todos os anos o parâmetro nP/V foi menor, tendo a mesma ocorrência em 2014 com P/nV.

Tabela 2: Serie B

\begin{tabular}{lllllll}
\hline & $\mathrm{P} / \mathrm{V}$ & $\mathrm{P} / \mathrm{nV}$ & $\mathrm{nP} / \mathrm{V}$ & $\mathrm{nP} / \mathrm{nV}$ & Qui-quadrado & valor de $\mathrm{p}$ \\
\hline $\mathbf{2 0 1 1}$ & 232 & 110 & 35 & 307 & 238,419 & $0,001^{*}$ \\
$\mathbf{2 0 1 2}$ & 211 & 128 & 129 & 215 & 41,809 & $0,001^{*}$ \\
$\mathbf{2 0 1 3}$ & 249 & 101 & 47 & 303 & 238,851 & $0,001^{*}$ \\
$\mathbf{2 0 1 4}$ & 224 & 126 & 132 & 218 & 48,38 & $0,001^{*}$ \\
\hline
\end{tabular}

Legenda: $\mathrm{P}=$ Marcou primeiro gol; $\mathrm{nP}=$ Não marcou primeiro gol; $\mathrm{V}=$ Venceu; $\mathrm{nV}=$ Não Venceu.

Observam-se na Tabela 2 os resultados nos jogos do Campeonato Brasileiro da Série B dos anos de 2011 a 2014. Evidencia-se uma associação significativa entre marcar o primeiro gol e sair vencedor na partida em todos os anos investigados ( $p=0,001$ em todos os anos). Desta forma, observa-se que de acordo com os valores da tabela assim como apresentado nos dados da série $\mathrm{A}$, de 2011 a 2013 o parâmetro $\mathrm{nP} / \mathrm{nV}$ possuiu maior ocorrência sendo superado apenas no ano de 2014 pelo parâmetro P/V. Tendo como menor ocorrência na Tabela 2 o parâmetro nP/nV nos anos de 2011 e 2013 e nos anos de 2012 e 2014 o parâmetro $\mathrm{P} / \mathrm{nV}$. 


\section{Discussão}

O presente estudo teve como objetivo investigar a associação entre a marcação do primeiro gol e o resultado final de partidas do Campeonato Brasileiro séries A e B nas temporadas 2011, 2012, 2013 e 2014. Os resultados encontrados apontaram uma associação significativa entre se marcar o primeiro gol e vencer a partida, para todos os Campeonatos Brasileiros analisados séries A e B.

$\mathrm{Na}$ literatura os estudos encontrados apresentam resultados semelhantes aos achados do presente aporte. Apesar da grande importância e investigação do gol no jogo de futebol (DAVID et al., 2013) poucos estudos relacionaram a marcação do primeiro gol e o resultado final da partida. No futsal Leite (2014) encontrou uma associação de 71,15\% entre se marcar o primeiro gol e vencer a partida durante Copa do Mundo de 2012. Já no futebol Michailidis et al. (2013) analisaram a Copa dos Campeões Europeus, quanto à marcação do gol e o resultado final da partida e observaram uma associação com a vitória em 70,97\%. Em consonância a isso, analisando o futebol brasileiro observou-se uma associação significativa em se marcar o primeiro gol e vencer a partida $(p=0,001)$ nas duas principais divisões do futebol brasileiro, séries A e B.

O jogo de futebol se apresenta inserido em uma lógica complexa de acontecimentos previsíveis e imprevisíveis (GARGANTA, 2009), logo esses acontecimentos, como o gol têm implicações na forma como as equipes se organizam em função da configuração complexa da partida. No Futebol, diante da baixa quantidade de gols marcados, a defesa assume especial importância (VOGELBEIN et al., 2014). Assim, a desorganização do processo defensivo adversário criado pela marcação do primeiro gol da partida, se tornaria um pressuposto vantajoso, por meio do qual se exploram ações técnico-táticas individuais e coletivas, visando concretizar o objetivo final. Observa-se que o placar momentâneo do jogo interfere no comportamento dos jogadores (BRADLEY et al., 2014; LAGO, 2009). Desta forma, as equipes que marcam o $1^{\circ} \mathrm{gol}$ eventualmente dispõem da vantagem de explorar possíveis desajustes da defesa adversária. Assim as equipes que marcam o primeiro gol da partida teriam vantagens em relação ao seu objetivo final, a vitória.

A análise do gol no futebol no que tange ao momento de marcação e status da partida se torna importante para entender o resultado final da partida de futebol, seguindo uma lógica de acontecimentos complexa. Desta forma, as equipes devem saber atacar, defender e passar de um momento para o outro (ataque-defesa) sem abdicar do equilíbrio coletivo e os objetivos da equipe, mantendo assim o Modelo de Jogo (SILVA, 2008). O êxito de ambas as fases passa por uma coordenação das ações dos jogadores reguladas pelos princípios gerais e específicos (CLEMENTE et al., 2014; COSTA et al., 2009). Portanto o processo de ensino-aprendizagem-treinamento do futebol deve incidir sob a coordenação das acões coletivas e individuais e a manutenção da organização e equilibrio nos momentos do jogo, 
mesmo mediante os acontecimentos que possam causar desordem, como sofrer ou marcar o primeiro gol.

\section{Considerações Finais}

Conclui-se haver associação significativa e positiva entre a marcação do primeiro gol e a obtenção da vitória em partidas das séries A e B do campeonato brasileiro. Tal associação indica que marcar o primeiro gol eleva as chances de vitória das equipes nacionais de futebol, resultado consonante com o reportado em outras ligas ao redor do mundo.

$\mathrm{Na}$ medida em que o jogo de futebol se caracteriza por uma reduzida quantidade de pontos (ou gols) comparativamente aos demais jogos esportivos coletivos, era esperada uma associação entre a marcação do primeiro gol e a vitória nos jogos do campeonato brasileiro. Contudo, a magnitude desta associação demonstrada neste estudo repercute na necessidade de comissões técnicas estabelecerem padrões técnico-táticos capazes de utilizar o potencial impacto positivo da marcação do primeiro gol a seu favor e reverter o potencial impacto negativo associado à marcação do gol pela equipe adversária. Por um lado, o estabelecimento de padrões de jogo orientados para explorar eventuais desajustes defensivos emergentes a partir da busca pelo gol da equipe momentaneamente atrás no placar pode auxiliar na marcação de mais gols e, consequentemente, ampliar as possibilidades de vitória. Por outro lado, o estabelecimento de padrões de jogo a partir de referências funcionais e espaciais amplamente conhecidas pelos atletas da equipe pode inibir potenciais impactos negativos da marcação do gol pela equipe adversária e, consequentemente, auxiliar a equipe a recuperar-se no placar. 


\section{Referências}

AGUIAR, M.; BOTELHO, G.; LAGO, C.; MAÇAS, V.; SAMPAIO, J.A review on the effects of soccer small-sided games. Journal of Human Kinetics, 33(1), 103-113. 2012.

ARAÚJO, D.; DAVIDS, K.; HRISTOVSKI, R. The ecological dynamics of decision making in sport. Psychology of sport and exercise, v. 7, p. 653-676, 2006.

BRADLEY, P.; LAGO-PEÑAS, C.; REY, E.; SAMPAIO, J. The influence of situational variables on ball possession in the english premier league. Journal of Sports Sciences, v. 32, n. 20, p. 1867-1873, 2014.

CARLING, C.; WILLIAMS, A; REILLY, T. Handbook of Soccer Match Analysis, London: Routledge. 2002.

CARLING, C.; WILLIAMS, M.; REILLY, T. Handbook of Soccer Match Analysis: A Systematic Approach to Improving Performance. London: Routledge. 2005.

CASTELLANO, J.; CASAMICHANA, D. What are the differences between first and second divisions of spanish football teams? International Journal of Performance Analysis in Sport, v. 15, p. 135-146, 2015.

CLEMENTE, F. M.; MARTINS, F. M. L.; MENDES, R. S.; FIGUEIREDO, A. J. A systemic overview of football game: The principles behind the game. Journal of Human Sport \& Exercise, v. 9, n. 2, p. 656-667, 2014.

COSTA, I. T.; GARGANTA, J. M.; GRECO, P. J.; MESQUITA, I. Princípios táticos do jogo de futebol: Conceitos e aplicação. Revista Motriz, v. 15, 2009.

DAVID, G. B.; PICANÇO, L. M.; REICHERT, F. F. Análise de fatores determinantes do gol no futsal feminino. Revista Brasileira de Futsal e Futebol, v. 6, n. 19, 2013.

GARGANTA, J. Modelação tática do jogo de futebol: estudo da organização da fase ofensiva em equipas de alto rendimento. (Doutorado), Universidade do Porto. 1997.

GARGANTA, J. M. Trends of tactical performance analysis in team sports: Bridging the gap between research, training and competition. Revista Portuguesa de Ciência do Desporto, v. 9, n. 1, p. 81-89, 2009.

GRECO, P. J.; Benda, R. N. Iniciação Esportiva Universal (Vol. 1). Belo Horizonte: Editora UFMG. 1998.

GOMES, A. C.; SOUZA, J. Futebol: treinamento desportivo de alto rendimento. São Paulo: Editora Artmed. 2008.

HUGNES,M.; BARTLETT, R. Performance indicators in performance analysis. Journal of Sports Sciences, 20(3), 738-754. 2002 
LAGO, C. The influence of match location, quality of opposition, and match status on possession strategies in professional association football. Journal of Sports Sciences, v. 27, n. 13, p. 1463-1469, 2009.

LUHTANEN, P.; BELINSKIJ, A.; HÄYRINEN, M.; VÄNTTINEN, T.A comparative tournament analysis between EURO 1996 and 2000in soccer. International Journal of Performance Analysis in Sport, 1(1), 74 - 82. 2001.

LEITE, W. S. S. Relação entre o primeiro gol e o resultado final do jogo de futsal na Copa do Mundo de 2012. Revista Brasileira de Futsal e Futebol, São Paulo. v.6. n.19. p.32-36. 2014.

MICHAILIDIS, Y.; MICHAILIDIS, C.; PRIMPA, E. Analysis of goals scored in European Championship 2012. Journal of Human Sport \& Exercise, vol. 8. Núm. 2. p.367-375. 2013

MONLINUEVO, J S.; BERMEJO, J P. El efecto de marcar primero y la ventaja de jugar en casa en la liga de fútbol y en la liga de fútbol sala de España. Revista de Psicología del Deporte, vol. 21, núm. 2, pp. 301-308. 2012.

SILVA, M. O desenvolvimento do jogar, segundo a Periodização Táctica. Editora McSports. 2008

THOMAS, J.; NELSON, J. K.; SILVERMAN, S. J. Métodos de pesquisa em atividade física. Porto Alegre: Artmed, 2012.

VOGELBEIN, M.; NOPP, S.; HÖKELMANN, A. Defensive transition in soccer - are prompt possession regains a measure of success? A quantitative analysis of german fußball-bundesliga 2010/2011. Journal of Sports Sciences, v. 32, n. 11, p. 1076-1083. 2014.

YIANNAKOS, A; ARAMATAS; V. Evaluation of The Goal Scoring Patterns in European Championship in Portugal 2004. International Journal of Perfomance Analysis in Sport. 11, 178 - 188. University of Wales Institute, Cardiff. 2006. 


\title{
The influence of scoring first in the result of matches of the series $A$ and B Brazilian championship
}

\begin{abstract}
This article aimed to investigate the influence of scoring first in the result of Brazilian championship Serie A and B matches. The association between result and scoring first was analyzed from 2011 to 2014 Serie A and B championships. It was observed a significant association between scoring first and the result in all Serie A championships. Similar results were found in the Serie B, revealing the importance of scoring first to obtain victories in football matches. There is a significant association between scoring first and obtaining wins in the Brazilian championship.
\end{abstract}

Key-words: Football; Situational variables; Brazilian Championship.

\section{La influencia del primer gol en el resultado final de los partidos del Campeonato Brasileiro series A y B}

\section{Resumen}

El objetivo del estudio fue analizar la influencia de marcar el primer gol en el resultado final de juegos en el Campeonato Brasilero de futbol series A y B. Se analizaron datos durante el periodo de 2011 a 2014. Los resultados evidenciaron asociación significativa entre marcar el primero gol y vencer el juego en las temporadas de la serie $A$ y $B$, revelando la importancia de esta variable para sí obtener la victoria en el futbol. Se concluye que existe una asociación significativa entre marcar el primer gol y obtener victorias en el campeonato brasileiro.

Palabras-Clave: Fútbol; Variables situacionales; Campeonato Brasileño 